\title{
Caffeine improves bladder function in diabetic rats via a neuroprotective effect
}

\author{
JUN XUE, YADONG LIU, SICHONG ZHANG, LIUCHENG DING, \\ BAIXIN SHEN, YUNPENG SHAO and ZHONGQING WEI
}

\begin{abstract}
Department of Urology, The Second Affiliated Hospital of Nanjing Medical University, Nanjing, Jiangsu 210011, P.R. China
\end{abstract}
Received April 30, 2020; Accepted February 18, 2021

DOI: $10.3892 /$ etm.2021.9932

\begin{abstract}
Diabetic cystopathy (DCP) is one of the most common complications of diabetes mellitus (DM). A previous study reported that caffeine may improve bladder dysfunction in rats with DM. The aim of the present study was to investigate the mechanisms behind the capacity for caffeine to improve bladder function in rats with DM. Sprague Dawley rats were divided into four groups: control, caffeine, DM and DM plus caffeine treatment (DM + caffeine). Bladder function was measured by urodynamic analyses. The levels of nerve growth factor (NGF), brain-derived neurotrophic factor (BDNF) and calcitonin gene-related peptide (CGRP) in the bladder tissue were detected by ELISA. Apoptosis in the dorsal root ganglion (DRG) was detected by terminal deoxynucleotidyl transferase-mediated dUTP nick end labeling assay. The expression levels of B-cell lymphoma-2 (Bcl-2), Bcl-2-associated X protein (Bax), caspase-3, cleaved caspase-3, caspase-9 and cleaved caspase -9 proteins in the DRG were detected by western blotting. Following treatment with caffeine, the urination time and micturition interval of rats with DM were improved, the bladder wet weight was decreased, and the maximum voiding pressure was increased. Relative to that in the DM group, the expression levels of NGF, BDNF and CGRP in the bladder tissue of DM + caffeine rats increased; cellular apoptosis in the DRG of DM + caffeine rates decreased; and the expression levels of Bcl-2, Bax, cleaved caspase- 3 and cleaved caspase- 9 proteins in the DRG of DM + caffeine rats were restored to a certain extent. In conclusion, caffeine promotes bladder function in rats with DM through a protective effect on DRG.
\end{abstract}

Correspondence to: Professor Zhongqing Wei, Department of Urology, The Second Affiliated Hospital of Nanjing Medical University, 121 Jiangjiayuan Road, Nanjing, Jiangsu 210011, P.R. China

E-mail: njuweizq@163.com

Key words: caffeine, bladder function, diabetes mellitus, dorsal root ganglion

\section{Introduction}

In recent years, with ongoing lifestyle and diet changes, population aging, and the rise in rates of obesity, the incidence of diabetes mellitus (DM) is increasing at an alarming rate (1). One of the common complications of DM is diabetic cystopathy (DCP) and, even in patients with stable blood glucose control, the incidence of DCP is as high as 25\% (2). DCP is insidious and has an asymptomatic progressive development, which is easily ignored by clinicians and diabetic patients before ultimately causing chronic urinary retention and urinary incontinence. DCP mainly manifests in affected patients as reduced bladder contractility and decreased urination. Upon progressing to the end stage, the disease is irreversible and is accompanied by urinary tract infections and reflux hydronephrosis, which seriously affect the quality of life of patients and may even be life-threatening (3).

The exact pathogenesis of DCP remains inadequately understood to date and the condition is mainly attributed to factors, including peripheral autonomic neuropathy and impaired detrusor function of the bladder caused by DM. An increasing number of studies have suggested that certain signaling pathways affiliated with neurotransmitter-receptor binding serve an important role in elements of DCP, including the levels of peptide energy, nitrogen energy, sputum energy, serotonin, endothelin, gamma-aminobutyric acid and prostaglandins. Changes in the levels or activity of corresponding neurotransmitters and receptors may trigger bladder urethral dysfunction.

At present, although different conventional medical treatments are available for DCP, the problem of the high recurrence rate among patients cannot be satisfactorily solved. Cystostomy is considered an effective treatment for cystopathy but it seriously affects the quality of life of patients (4). It is generally believed that medical nutrition therapy is an important part of DM management (5). Previous guidelines and research have focused on the type and amount of carbohydrates consumed. However, how other food ingredients, including caffeine, affect DM remains unknown. Caffeine is the main ingredient in coffee and tea, which are consumed daily by numerous people. The association between caffeine and human health has long been of concern to scientists. A recent study suggested that caffeine may improve bladder function by promoting increased expression levels of nerve growth factor (NGF) and 
c-fos, improving bladder contractility, and repairing damaged bladder nerves (6), while our previous study reported that caffeine may improve bladder dysfunction in rats with DM (7). However, the underlying mechanism of this remains unclear. Another previous study has also indicated that calcitonin gene-related peptides (CGRP) are the main transmitters affiliated with bladder sensory nerves; specifically, the content of CGRP in the bladder wall of diabetic rats, particularly in the submucosal plexus and the CGRP nerve distribution, were significantly decreased, suggesting that CGRP serves a key role in the development of DCP (8).

Therefore, we hypothesized in the present study that caffeine may improve bladder function in rats with DM by protecting bladder sensory neurons and promoting the release of related neurotransmitters.

\section{Materials and methods}

Animal models. The present study was approved by the Ethical Committee on Animal Experiment Committee of Nanjing Medical University (Nanjing, China). The animal models were prepared using a protocol described previously by WenBo et al (9). Female Sprague Dawley (SD) rats $(n=64$; weight, 200-220 g) were purchased from Nanjing Medical University's Animal Experiment Center. All rats were housed under similar conditions (temperature, $22 \pm 2^{\circ} \mathrm{C}$; relative humidity, 50 $\pm 5 \%$; light/dark cycle, 12-h) with free access for food and water. A total of 40 rats were intraperitoneally injected with $60 \mathrm{mg} / \mathrm{kg}$ streptozotocin (STZ) (Sigma-Aldrich; Merck $\mathrm{KGaA}$ ), while the other 24 rats were intraperitoneally injected with the same volume of citric acid buffer. A level of fasting blood glucose (FBG) measured three days after STZ injection of $>16.7 \mathrm{mmol} / \mathrm{l}$ was considered to indicate that the DM model had been successfully induced. In total, $31 \mathrm{DM}$ rats were successfully induced, constituting $77.5 \%$ of the total study population. Next, 24 diabetic rats were stratified as the DM and DM plus caffeine treatment (DM + caffeine) groups ( $\mathrm{n}=12$ each), while $24 \mathrm{STZ}$-untreated rats were divided into the control and caffeine groups $(n=12$ each). The rats in the DM + caffeine and caffeine groups were treated with caffeine by oral gavage $(10 \mathrm{mg} / \mathrm{kg} / \mathrm{day})$ for 16 weeks. The urination time, micturition interval, bladder wet weight and maximum voiding pressure of rats were used to measure bladder function as reported in our previous study (10). Next, the rats were sacrificed and the lumbosacral-segment dorsal root ganglion (DRG) and bladder were prepared for analysis.

Hematoxylin and eosin $(H \& E)$ staining for the DRG tissues. After the rats were sacrificed by being intraperitoneally injected with $2 \%$ pentobarbital sodium $(180 \mathrm{mg} / \mathrm{kg})$, the spinal canal was opened posteriorly to expose the spinal cord and the posterior roots of the spinal nerves, the white and bright nodule on the posterior root of the spinal nerve near the intervertebral foramen was the DRG. DRG specimens were fixed with $10 \%$ neutral formalin at room temperature for $4 \mathrm{~h}$, routinely dehydrated, embedded, and cut into 4- $\mu$ m-thick sections for H\&E staining at room temperature for $8 \mathrm{~min}$. The result is shown in Fig. S1.

ELISA. NGF, brain-derived neurotrophic factor (BDNF), and CGRP levels in bladder tissue were measured using an ELISA kit for rats; specifically, the NGF ELISA kit (cat. no. ab193736; Abcam), BDNF ELISA kit (cat. no. ab213899; Abcam) or CGRP ELISA kit (cat. no. 589001; Cayman Chemical Company), according to the manufacturer's protocols. In brief, total proteins were extracted from the bladder using lysis buffer (50 mM Tris- $\mathrm{HCl}$, pH 7.5, $150 \mathrm{mM} \mathrm{NaCl}, 1 \mathrm{mM}$ EDTA, $1 \%$ NP-40, $0.5 \%$ deoxycholic acid, $0.1 \%$ SDS, $10 \mu \mathrm{l} / \mathrm{ml}$ protease inhibitor cocktail and $1 \mathrm{mM}$ PMSF) and the supernatants were collected by centrifuging at $4^{\circ} \mathrm{C}$ at $12,000 \mathrm{x}$ for $15 \mathrm{~min}$. Subsequently, 96-well microplates were coated with $100 \mu \mathrm{l}$ biotinylated primary antibodies mixed with $100 \mu$ l EIA buffer provided in the kit, plus $100 \mu \mathrm{l}$ standard and sample aliquots. Plates were incubated for $2 \mathrm{~h}$ at $30^{\circ} \mathrm{C}$, followed by aspiration of the samples and subsequent washing of them three times with wash buffer. Next, $100 \mu$ l solution of streptavidin-horseradish peroxidase conjugate was added to each well and incubated for $30 \mathrm{~min}$ at $30^{\circ} \mathrm{C}$, prior to washed again. Thereafter, $100 \mu \mathrm{l}$ substrate solution provided in the kits was added to each well and the plates were incubated for $30 \mathrm{~min}$ at $30^{\circ} \mathrm{C}$. The optical density values were read at $450 \mathrm{~nm}$ using a Biotek Synergy 2 plate reader (BioTek Instruments, Inc.).

Terminal deoxynucleotidyl transferase-mediated dUTP nick end labeling (TUNEL) assay. The apoptosis in DRG was measured using a TUNEL kit for rats (cat. no. C1086; Beyotime Institute of Biotechnology,) according to the manufacturer's protocols. Briefly, DRG specimens were fixed with $10 \%$ neutral formalin at room temperature for $4 \mathrm{~h}$, routinely dehydrated with an ascending ethanol gradient and xylene, embedded with paraffin, and cut into $4-\mu \mathrm{m}$-thick sections for TUNEL assay. The section was incubated with $100 \mu 1$ TUNEL reagent ( $5 \mu 1 \mathrm{TdT}$ enzyme, $45 \mu$ l fluorescent labeling solution, $50 \mu \mathrm{l}$ TUNEL test solution) in the dark at $37^{\circ} \mathrm{C}$ for $60 \mathrm{~min}$. Cell nuclei were counter-stained with Hoechst 33342 (1:2,000, Thermo Fisher Scientific, Inc.) for $30 \mathrm{~min}$ at room temperature. The sections were observed and photographed with a fluorescent microscope (Leica Microsystems $\mathrm{GmbH}$ ) at x400 magnification. Under the microscope, six fields were randomly selected to count the number of apoptotic cells for each section.

Western blot analysis. Western blot analysis was applied to determine the expression levels of B-cell lymphoma-2 (Bcl-2), Bcl-2-associated X protein (Bax), caspase-3, cleaved caspase-3, caspase- 9 and cleaved caspase- 9 proteins in DRG tissues. Tissues were homogenized in RIPA lysis buffer (Thermo Fisher Scientific, Inc.) with protease inhibitor cocktail (Roche Diagnostics), before the supernatants were collected by centrifuging at $4^{\circ} \mathrm{C}$ at $12,000 \mathrm{x}$ g for $15 \mathrm{~min}$. The total protein was quantified by UV spectrophotometry and $50 \mu \mathrm{g}$ protein was separated by $12 \%$ SDS-PAGE. Next, they were transferred onto the nitrocellulose membrane (EMD Millipore). The transferred membrane was washed with rinse buffer, prior to being incubated with blocking buffer (5\% skimmed milk in rinse buffer) for $30 \mathrm{~min}$ at room temperature. Subsequently, the membrane was incubated with the following primary antibodies for $4 \mathrm{~h}$ at room temperature: rabbit anti-Bcl-2 (cat. no. ab194583; dilution, 1:800; Abcam), rabbit anti-Bax (cat. no. ab32503; dilution, 1:800; Abcam), rabbit anti-cleaved caspase-3 (cat. no. ab214430; dilution, 
Table I. Parameters in the animals after 16 weeks.

\begin{tabular}{lccrr}
\hline Parameters & Control & Caffeine & DM & DM + caffeine \\
\hline Body weight, g & $303.92 \pm 10.52$ & $302.92 \pm 10.40$ & $190.33 \pm 8.02^{\mathrm{a}, \mathrm{b}}$ & $215.00 \pm 22.54^{\mathrm{a}-\mathrm{c}}$ \\
FBG, mmol/l & $4.60 \pm 1.27$ & $4.59 \pm 1.11$ & $25.33 \pm 3.07^{\mathrm{a}, \mathrm{b}}$ & $23.13 \pm 5.96^{\mathrm{a}, \mathrm{b}}$ \\
Bladder wet weight, g & $0.20 \pm 0.02$ & $0.20 \pm 0.03$ & $0.40 \pm 0.07^{\mathrm{a}, \mathrm{b}}$ & $0.28 \pm 0.04^{\mathrm{a}-\mathrm{c}}$ \\
Urination time, sec & $18.33 \pm 2.42$ & $18.17 \pm 2.29$ & $25.92 \pm 3.73^{\mathrm{a}, \mathrm{b}}$ & $22.08 \pm 3.12^{\mathrm{a}-\mathrm{c}}$ \\
Micturition interval, sec & $40.67 \pm 3.87$ & $41.50 \pm 3.66$ & $236.33 \pm 63.94^{\mathrm{a}, \mathrm{b}}$ & $73.67 \pm 13.18^{\mathrm{c}}$ \\
Maximum voiding pressure, $\mathrm{mmHg}$ & $41.00 \pm 7.71$ & $45.67 \pm 6.54$ & $15.58 \pm 4.50^{\mathrm{a}, \mathrm{b}}$ & $29.42 \pm 6.26^{\mathrm{a}-\mathrm{c}}$ \\
\hline
\end{tabular}

${ }^{\mathrm{a}} \mathrm{P}<0.05$ vs. control group; ${ }^{\mathrm{b}} \mathrm{P}<0.05$ vs. caffeine group; ${ }^{\mathrm{c}} \mathrm{P}<0.05$ vs. DM group. DM, diabetes mellitus; FBG, fasting blood glucose.

1:1,000; Abcam), rabbit anti-caspase-3 (cat. no. ab184787; dilution, 1:1,000; Abcam), rabbit anti-cleaved caspase-9 (cat. no. 20750; dilution, 1:1,000; Cell Signaling Technology, Inc.), rabbit anti-caspase-9 (cat. no. ab184786; dilution, 1:1,000; Abcam) and mouse anti- $\beta$-actin (cat. no. A1978; dilution, 1:5,000; Sigma-Aldrich; Merck KGaA). Next, each membrane was washed with rinse buffer and incubated with the following secondary antibodies for $1.5 \mathrm{~h}$ at room temperature: IRDye 680-conjugated affinity-purified goat anti-mouse immunoglobulin G (cat. no. 610-144-121; dilution, 1:5,000; Rockland Immunochemicals)and IRDye 800 -conjugated affinity-purified goat anti-rabbit immunoglobulin G (cat. no. 611-145-122; dilution, 1:5,000; Rockland Immunochemicals). The results were visualized using the Odyssey laser scanning system (LI-COR Biosciences). The relative quantity of the protein bands was determined using the Odyssey version 3.0 software program (LI-COR Biosciences).

Statistical analysis. The data obtained in the present study were processed and analyzed using the SPSS version 21.0 software program (IBM Corp.). The measurement data are expressed as the mean \pm standard deviation. Differences between groups were estimated by one-way analysis of variance and compared using the Tukey's post hoc test. $\mathrm{P}<0.05$ was considered to indicate a statistically significant difference.

\section{Results}

General condition of the rats and urinary function of the bladder. As mentioned previously, 40 rats were prepared for the DM model with STZ and, of these, 31 rats were successfully induced for a success rate of $77.50 \%$. There was no significant difference in body weight between the control and caffeine groups $(\mathrm{P}>0.05)$. However, compared with that in the control and caffeine groups, the body weights of the rats in the DM and DM+caffeine groups were significantly decreased, with the decrease being more notable in the DM group, and the variations among the groups were statistically significant $(\mathrm{P}<0.05)$. There was no significant difference in FBG between the control and caffeine groups $(\mathrm{P}>0.05)$ and no significant difference in FBG between the DM and DM+caffeine groups $(\mathrm{P}>0.05)$. Compared with that in the control and caffeine groups, the bladder wet weight of the rats in the DM and DM+caffeine groups was significantly increased, with the bladder wet weight in the DM+caffeine group being lower than that in the DM group $(\mathrm{P}<0.05)$. By contrast, there was no significant difference in the urinary function between the control and caffeine groups $(\mathrm{P}>0.05)$. Compared with that in the control and caffeine groups, urinary function in the DM and DM+caffeine groups was significantly decreased, but the urinary function of the DM+caffeine group was significantly better than that of DM group $(\mathrm{P}<0.05)$. The results are presented in Table I.

$N G F, B D N F$ and CGRP levels in the bladder tissue. ELISA results demonstrated that there were no significant differences in the levels of NGF, BDNF and CGRP in the bladder tissues between the control and caffeine groups $(\mathrm{P}>0.05)$. Compared with that in the control and caffeine groups, the levels of NGF, BDNF and CGRP in the bladder tissues of the $\mathrm{DM}$ and DM+caffeine groups were significantly decreased, with the levels of NGF, BDNF and CGRP in the bladder tissue of the DM+caffeine group being higher than those in the DM group and the differences being statistically significant $(\mathrm{P}<0.05$; Fig. 1).

Cellular apoptosis in the DRG. TUNEL assay demonstrated that there were almost no apoptotic cells present in the DRG in the control and caffeine groups, while more apoptotic cells were observed in the DRG in the DM and DM+caffeine groups. Furthermore, the apoptotic cell count in the DRG in the DM+caffeine group was less than that in the DM group and all differences were statistically significant ( $\mathrm{P}<0.05$; Fig. 2).

Expression of Bcl-2, Bax, caspase-3, cleaved caspase-3, caspase-9 and cleaved caspase-9 proteins in the DRG. Western blot analysis demonstrated that there was no significant difference in the expression of Bcl-2 protein in the DRG between the control and caffeine groups $(\mathrm{P}>0.05)$. Compared with that in the control and caffeine groups, the relative expression levels of Bcl-2 protein in the DRGs of the DM and $\mathrm{DM}+$ caffeine groups were significantly decreased, while the expression level of $\mathrm{Bcl}-2$ protein in the $\mathrm{DM}+$ caffeine group was higher than that in the DM group and the difference was statistically significant ( $\mathrm{P}<0.05$; Fig. 3A).

The results of western blotting demonstrated that the expression levels of Bax, cleaved caspase- 3 and cleaved caspase- 9 proteins in the DRGs of the DM group and $\mathrm{DM}+$ caffeine group were higher than those in the control and caffeine groups and all differences were statistically 
A

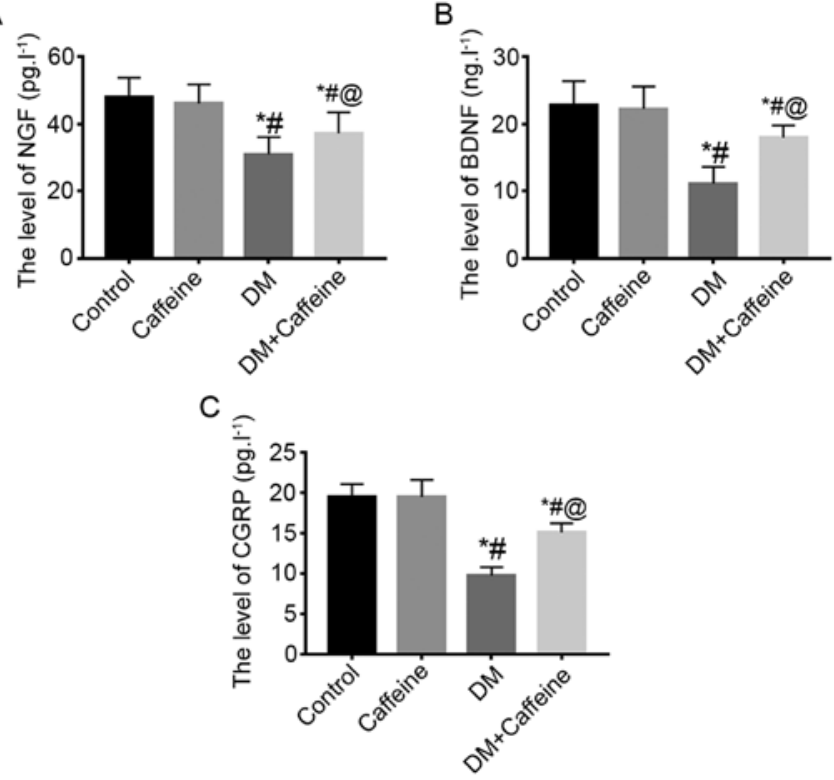

Figure 1. NGF, BDNF and CGRP levels in the bladder tissue. The levels of (A) NGF, (B) BDNF and (C) CGRP in rat bladders were detected by ELISA. ${ }^{*} \mathrm{P}<0.05$ vs. control; ${ }^{\sharp} \mathrm{P}<0.05$ vs. caffeine group; ${ }^{\circledR} \mathrm{P}<0.05$ vs. $\mathrm{DM}$ group. NGF, nerve growth factor; BDNF, brain-derived neurotrophic factor; CGRP, calcitonin gene-related peptide; DM, diabetes mellitus.

significant $(\mathrm{P}<0.05)$. Furthermore, the levels of Bax, cleaved caspase- 3 and cleaved caspase- 9 proteins in the DRGs of the $\mathrm{DM}+$ caffeine group were significantly lower than those in the DM group $(\mathrm{P}<0.05)$. The differences of caspase-3 and caspase-9 protein levels among groups were not statistically significant ( $\mathrm{P}>0.05$; Fig. 3B).

\section{Discussion}

Caffeine is a methylxanthine and acts as an agonist of the sarcoplasmic reticulum RyR receptor in the cell, regulating RyR receptor-mediated calcium release and mitochondria adenosine triphosphate production, thereby exerting a biological function. In the present study, a DM rat model was successfully prepared using STZ, prior to the DM rats being treated with caffeine for 16 weeks. The results demonstrated that bladder-function parameters, including urination time, micturition interval and maximum voiding pressure, were significantly improved in DM rats. Furthermore, the wet weight of the bladder in treated DM rats was markedly decreased. These findings suggested that caffeine may effectively improve the bladder function of DM rats. Additionally, the results of FBG analysis indicated that caffeine did not significantly decrease the level of FBG in the DM rats, suggesting that caffeine may have no impact on decreasing the blood glucose level and supporting the hypothesis that its pharmacological effect may be mainly associated with the target cells.

NGF is a member of the neurotrophic factor family and is found primarily in sympathetic, partial sensory nerves and distributed target organs. Previous studies have demonstrated that NGF is associated with the pathogenesis of bladder dysfunction caused by multiple etiologies (11-13). NGF expression levels in tissues have been significantly decreased in clinical animal models and the promotion of

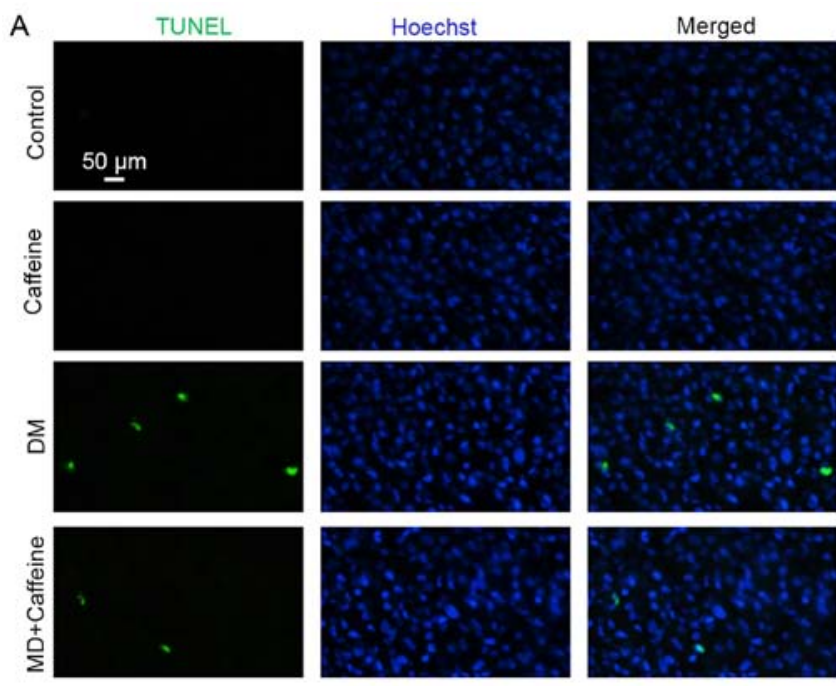

B

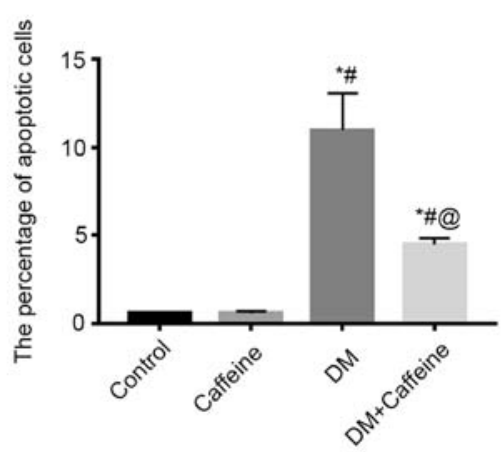

Figure 2. Cellular apoptosis in the DRG. (A) TUNEL assay was used to detect apoptotic cells in rat DRGs. (B) A statistical graph of the percentage of apoptotic cells in the DRG tissue of each group. ${ }^{\text {" }} \mathrm{P}<0.05$ vs. control group; ${ }^{~} \mathrm{P}<0.05$ vs. caffeine group; ${ }^{\circledR} \mathrm{P}<0.05$ vs. DM group. Scale bar, $50 \mu \mathrm{m}$. DRG, dorsal root ganglion; DM, diabetes mellitus.

NGF expression may improve the function of the bladder to a certain extent (11-13). Additionally, BDNF is another important neurotrophic factor. Its amino acid sequence is similar to that of NGF and is widely found in the nervous system and corresponding target organs. BDNF-overexpression is associated with an overactive bladder (14). Certain treatments may improve bladder function by promoting the recovery of the BDNF expression level (15).

Finally, CGRP is produced by sensory neurons. Once released from the cells, CGRP initiates a biological response by binding to a specific CGRP receptor located on cell surfaces and serves a key role in a variety of physiological and pathological processes, including cell proliferation, differentiation, apoptosis, and inflammatory and immune responses (16). It was reported that CGRP was the main transmitter of bladder sensory nerves (8). Elsewhere, the content of CGRP in the bladder walls of diabetic rats, particularly in the submucosal plexus, was significantly decreased and CGRP nerve distribution was also significantly decreased, suggesting that CGRP serves a key role in the development of DCP (8). The results of the present study indicated that the expression levels of NGF, BDNF and CGRP were significantly decreased in the bladder tissues of DM rats. After 16 weeks of caffeine treatment, the expression levels of NGF, BDNF and CGRP were restored to 


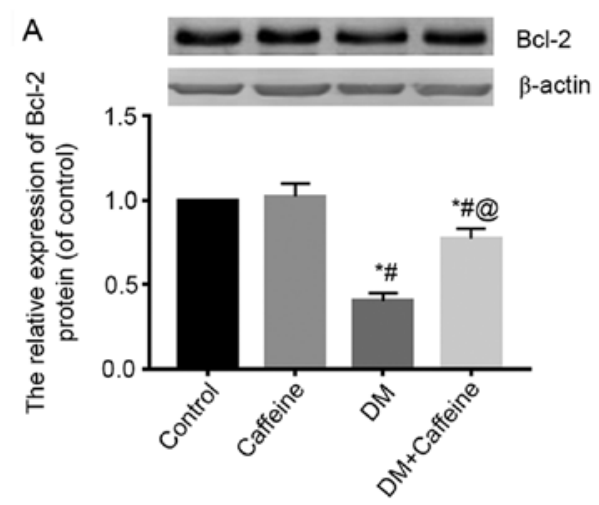

$B$
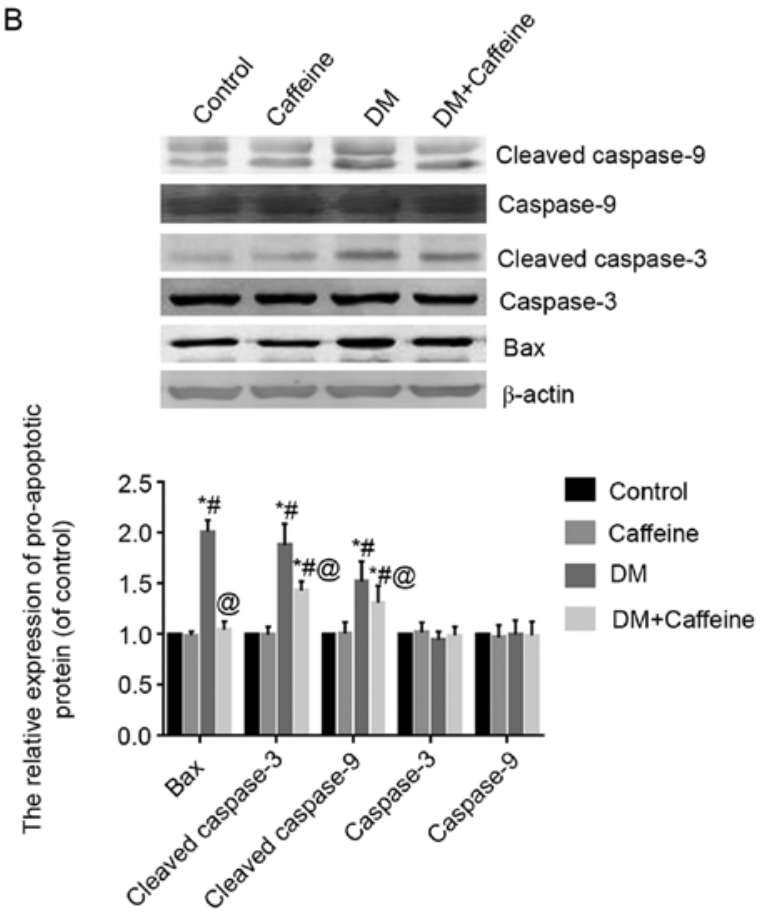

Figure 3. Expression levels of Bcl-2, Bax, caspase-3 and caspase-9 proteins in the DRG. (A) The expression level of Bcl-2 protein in rat bladders was detected by western blot analysis. (B) The expression levels of Bax, caspase-3, cleaved caspase- 3 , caspase- 9 and cleaved caspase- 9 proteins in rat bladders were detected by western blot analysis. ${ }^{*} \mathrm{P}<0.05$ vs. control group; ${ }^{*} \mathrm{P}<0.05$ vs. caffeine group; ${ }^{\circledR} \mathrm{P}<0.05$ vs. DM group. Bcl-2, B-cell lymphoma-2; Bax, Bcl-2-associated X protein; DRG, dorsal root ganglion; DM, diabetes mellitus.

a certain extent in the bladder tissues of DM rats but remained lower than those in the normal control rats. These results supported the idea that caffeine may promote the synthesis and release of neurotrophic factors in bladder tissues; however, the specific mechanism behind this concept requires further investigation. NGF, BDNF and CGRP have neurotrophic effects, which reduce the neuronal damage caused by high glucose by exerting a protective effect on DRG neurons, and may regulate certain biological signaling pathways to improve bladder function by acting on corresponding receptors on bladder smooth muscles $(15,16)$.

DRG is the main source of bladder sensory nerve fibers. Previous studies have demonstrated that, in the context of chronic hyperglycemia, neuron apoptosis and the number of autonomic nerves distributed in the bladder were decreased, further aggravating the level of damage to the bladder smooth muscle function $(17,18)$. The results of the present study revealed that apoptotic cells of the DRG in DM rats were significantly decreased following caffeine treatment, suggesting that caffeine has neuroprotective effects. This result may be associated with the increased expression of NGF, BDNF and CGRP triggered by caffeine. Several previous studies have reported that NGF, BDNF and CGRP may bind to their specific receptors, activate the related signaling pathways, and subsequently exert anti-apoptotic cytoprotective actions (19-24). There are numerous apoptotic proteins known to be involved in the process of apoptosis, among which the anti-apoptotic protein $B C L 2$, the pro-apoptotic protein $B A X$, caspase-3 and caspase- 9 have been heavily studied (25-28). The results of the present study demonstrated that the expression levels of Bax, cleaved caspase- 3 and cleaved caspase- 9 proteins in the DRG were increased in DM rats, while the expression level of the Bcl-2 protein was decreased. Following caffeine treatment, the expression levels of Bax, cleaved caspase- 3 , cleaved caspase- 9 and Bcl-2 proteins were partially restored. These results suggest that the effect of caffeine on protecting DRG cells from apoptosis is associated with the regulation of Bax, cleaved caspase- 3 , cleaved caspase- 9 and Bcl-2 expression levels.

There are certain limitations to the present study. At present, the majority of previous studies have focused on sensory neurons. However, the urinary bladder contractile function is regulated by sensory neurons, the motor neurons innervating the bladder, and the smooth muscle function. The bladder is controlled by two types of motor neurons, innervated by sympathetic and parasympathetic efferent nerves, which has highlighted certain difficulties to the related research, which may also be the reason for the lack of relevant literature. It may be necessary to design more sophisticated experiments to solve this problem. In addition, the mechanism by which caffeine attenuated the bladder hypertrophy was not sufficiently addressed in the present study. We hypothesize that CGRP may be associated with the mechanism by which caffeine attenuated the bladder hypertrophy. Finally, considering the impact on cells when digested into single cell suspension, flow cytometry detection was not performed in the present study, and the level of apoptosis was assessed using the percentage of the number of apoptotic cells to the number of total cells and the detected of related apoptotic proteins.

In summary, the results of the present study suggested that caffeine promotes bladder function in rats with DM through protective effects on the DRG, which may involve at least the signaling pathways associated with Bax, caspase-3, caspase-9 and Bcl-2.

\section{Acknowledgements}

Not applicable.

\section{Funding}

The present study was supported by National Natural Science Foundation of China (grant no. 81400758), Six Top Talent Fund of Jiangsu Province (grant no. 2014-wsw-12), and Nanjing Medical Science and Technology Development Project (grant no. YKK17210). 


\section{Availability of data and materials}

The datasets generated and/or analyzed during the current study are available in the Baidu Netdisk repository, https://pan. baidu.com/s/1IkHrw9skcDaNP71RtocS5A (code: puem).

\section{Authors' contributions}

ZW designed the study. JX, YL and SZ performed the experiment. LD, BS and YS performed the data analyses. JX and $\mathrm{ZW}$ wrote the manuscript. JX and ZW authenticated the raw data in this study. All authors reviewed and approved the final version of the manuscript.

\section{Ethics approval and consent to participate}

The present study was approved by the Ethical Committee on Animal Experiment Committee of Nanjing Medical University (Nanjing, China).

\section{Patient consent for publication}

Not applicable.

\section{Competing interests}

The authors declare that they have no competing interests.

\section{References}

1. Xu Y, Wang L, He J, Bi Y, Li M, Wang T, Wang L, Jiang Y, Dai M, Lu J, et al: Prevalence and control of diabetes in Chinese adults. JAMA 310: 948-959, 2013.

2. Yuan Z, Tang Z, He C and Tang W: Diabetic cystopathy: A review. J Diabetes 7: 442-447, 2015.

3. Arrellano-Valdez F, Urrutia-Osorio M, Arroyo C and Soto-Vega E: A comprehensive review of urologic complications in patients with diabetes. Springerplus 3: 549, 2014.

4. Feifer A and Corcos J: Contemporary role of suprapubic cystostomy in treatment of neuropathic bladder dysfunction in spinal cord injured patients. Neurourol Urodyn 27: 475-479, 2008.

5. Franz MJ, Bantle JP, Beebe CA, Brunzell JD, Chiasson JL, Garg A, Holzmeister LA, Hoogwerf B, Mayer-Davis E, Mooradian AD, et al: Evidence-based nutrition principles and recommendations for the treatment and prevention of diabetes and related complications. Diabetes Care 26 (Suppl 1): S51-S61, 2003.

6. Cho YS, Ko IG, Kim SE, Hwan L, Shin MS, Kim CJ, Kim SH, Jin JJ, Chung JY and Kim KH: Caffeine enhances micturition through neuronal activation in micturition centers. Mol Med Rep 10: 2931-2936, 2014.

7. Yi CR, Wei ZQ, Deng XL, Sun ZY, Li XR and Tian CG: Effects of coffee and caffeine on bladder dysfunction in streptozotocin-induced diabetic rats. Acta Pharmacol Sin 27: 1037-1043, 2006.

8. Langdale CL, Thor KB, Marson L and Burgard EC: Maintenance of bladder innervation in diabetes: A stereological study of streptozotocin-treated female rats. Auton Neurosci 185: 59-66, 2014.

9. WenBo W, Fei Z, YiHeng D, Wei W, TingMang Y, WenHao Z, QianRu L and HaiTao L: Human umbilical cord mesenchymal stem cells overexpressing nerve growth factor ameliorate diabetic cystopathy in rats. Neurochem Res 42: 3537-3547, 2017.

10. Liu YD, Zhang SC, Xue J, Wei ZQ, Shen BX and Ding LC: Caffeine improves bladder function in streptozotocin-induced diabetic rats. Neurourol Urodyn 38: 81-86, 2018.
11. Kashyap M, Pore S, Yoshimura N and Tyagi P: Constitutive expression Of NGF And P75(NTR) affected by bladder distension and NGF antisense treatment. Life Sci 148: 93-98, 2016.

12. Wada N, Shimizu T, Shimizu N, de Groat WC, Kanai AJ, Tyagi P, Kakizaki $\mathrm{H}$ and Yoshimura N: The effect of neutralization of nerve growth factor (NGF) on bladder and urethral dysfunction in mice with spinal cord injury. Neurourol Urodyn 37: 1889-1896, 2018.

13. Tonyali S, Ates D, Akbiyik F, Kankaya D, Baydar D and Ergen A: Urine nerve growth factor (NGF) level, bladder nerve staining and symptom/problem scores in patients with interstitial cystitis. Adv Clin Exp Med 27: 159-163, 2018.

14. Kashyap MP, Pore SK, de Groat WC, Chermansky CJ, Yoshimura $\mathrm{N}$ and Tyagi P: BDNF overexpression in the bladder induces neuronal changes to mediate bladder overactivity. Am J Physiol Renal Physiol 315: F45-F56, 2018.

15. Kashyap MP, Roberts C, Waseem M and Tyagi P: Drug targets in neurotrophin signaling in the central and peripheral nervous system. Mol Neurobiol 55: 6939-6955, 2018.

16. Parameswaran N, Disa J, Spielman WS, Brooks DP, Nambi P and Aiyar N: Activation of multiple mitogen-activated protein kinases by recombinant calcitonin gene-related peptide receptor. Eur J Pharmacol 389: 125-130, 2000.

17. Nirmal J, Tyagi P, Chuang YC, Lee WC, Yoshimura N, Huang CC, Rajaganapathy B and Chancellor MB: Functional and molecular characterization of hyposensitive underactive bladder tissue and urine in streptozotocin-induced diabetic rat. PLoS One 9: e102644, 2014.

18. Daneshgari F, Liu G, Birder L, Hanna-Mitchell AT and Chacko S: Diabetic bladder dysfunction: Current translational knowledge. J Urol 182 (Suppl 6): S18-S26, 2009.

19. Li R, Wu Y, Zou S, Wang X, Li Y, Xu K, Gong F, Liu Y, Wang J, Liao Y, et al: NGF attenuates high glucose-induced ER stress, preventing schwann cell apoptosis by activating the PI3K/Akt/GSK3 $\beta$ and ERK1/2 pathways. Neurochem Res 42: 3005-3018, 2017.

20. Sun Z, Hu W, Yin S, Lu X, Zuo W, Ge S and Xu Y: NGF protects against oxygen and glucose deprivation-induced oxidative stress and apoptosis by up-regulation of HO-1 through MEK/ERK pathway. Neurosci Lett 641: 8-14, 2017.

21. Cao J, Wu Y, Liu G and Li Z: Over-expression of BDNF inhibits angiotensin II-induced apoptosis of cardiomyocytes in SD rats. Xi Bao Yu Fen Zi Mian Yi Xue Za Zhi 34: 218-224, 2018 (In Chinese).

22. QiG,MiY,Wang Y,LiR,Huang S,LiX and LiuX:Neuroprotective action of tea polyphenols on oxidative stress-induced apoptosis through the activation of the TrkB/CREB/BDNF pathway and Keap1/Nrf2 signaling pathway in SH-SY5Y cells and mice brain. Food Funct 8: 4421-4432, 2017.

23. Ma YX, Guo Z and Sun T: CGRP inhibits norepinephrine induced apoptosis with restoration of $\mathrm{Bcl}-2 / \mathrm{Bax}$ in cultured cardiomyocytes of rat. Neurosci Lett 549: 130-134, 2013.

24. Yang JH, Zhang YQ and Guo Z: Endogenous CGRP protects retinal cells against stress induced apoptosis in rats. Neurosci Lett 501: 83-85, 2011.

25. Renault TT, Dejean LM and Manon S: A brewing understanding of the regulation of $\mathrm{Bax}$ function by $\mathrm{Bcl}-\mathrm{xL}$ and $\mathrm{Bcl}-2$. Mech Ageing Dev 161: 201-210, 2017.

26. Vogel MW: Cell death, Bcl-2, Bax, and the cerebellum. Cerebellum 1: 277-287, 2002.

27. Song L, Gao LN, Wang J, Thapa S, Li Y, Zhong XB, Zhao HW, Xiang XR, Zhang FG and Ji P: Stromal cell-derived factor-1 $\alpha$ alleviates calcium-sensing receptor activation-mediated ischemia/reperfusion injury by inhibiting caspase-3/caspase-9-induced cell apoptosis in rat free flaps. Biomed Res Int 2018: 8945850, 2018.

28. Lan T, Zhao H, Xiang B, Wang J and Liu Y: Suture compression induced midpalatal suture chondrocyte apoptosis with increased caspase-3, caspase-9, Bad, Bak, Bax and Bid expression. Biochem Biophys Res Commun 489: 179-186, 2017.

This work is licensed under a Creative Commons Attribution-NonCommercial-NoDerivatives 4.0 International (CC BY-NC-ND 4.0) License. 